\title{
The Initial Economic Burden of Femur Fractures on Informal Caregivers in Dar es Salaam, Tanzania
}

\section{Ericka P. von Kaeppler ${ }^{1 *}$, Erik J. Kramer ${ }^{2 *}$, Claire A. Donnelley ${ }^{1}$, Hao-Hua Wu ${ }^{1}$, Elliot Marseille ${ }^{4}$, Edmund Eliezer ${ }^{5}$, Heather J. Roberts ${ }^{1}$, David Shearer ${ }^{1}$, Saam Morshed}

\author{
1. Institute of Global Orthopaedics and Traumatology at the University of California San Francisco \\ 2. Yale University School of Medicine \\ 3. Health Strategies International \\ 4. Global Health Economics Consortium at the University of California San Francisco \\ 5. Muhimbili Orthopaedic Institute
}

Correspondence: David Shearer (davidshearer@icsf.edu)

\author{
Background \\ Femur fracture patients require significant in-hospital care. The burden incurred by caregivers of such patients amplifies the direct \\ costs of these injuries and remains unquantified. \\ Aim \\ Here we aim to establish the in-hospital economic burden faced by informal caregivers of femur fracture patients. \\ Methods: 70 unique caregivers for 46 femoral shaft fracture patients were interviewed. Incurred economic burden was determined by \\ the Human Capital Approach, using standardized income data to quantify productivity loss (in \$USD). Linear regression assessed the \\ relationship between caregiver burden and patient time-in-hospital. \\ Results \\ The average economic burden incurred was $\$ 149,9 \%$ of a caregiver's annual income and positively correlated with patient time in \\ hospital $(\mathrm{p}<0.01)$. \\ Conclusion \\ Caregivers of patients treated operatively for femur fractures lost a large portion of their annual income, and this loss increased with \\ patient time in hospital. These indirect costs of femur fracture treatment constitute an important component of the total injury \\ burden.
}

Keywords: Orthopaedic trauma; informal caregivers; economic burden; femur fracture; Tanzania

\section{Introduction}

Traumatic injuries are a growing global health concern and disproportionately affect low- and middle-income countries (LMICs). They contribute more to disability than HIV, tuberculosis, or malaria ${ }^{1-3}$. Road traffic accidents cause the majority of these injuries and lead to over 1.2 million deaths and up to 50 million non-fatal injuries annually ${ }^{3}$. Particularly in LMICs, femoral shaft fractures are a common result of road traffic accidents and represent a significant source of disability ${ }^{4,5}$. Since these injuries predominate in low-resource environments where there is minimal road traffic safety, patients who sustain femur fractures can incur tremendous financial burden — as much as $88 \%$ of their pre-injury income ${ }^{6}$. While studies have examined the effect of treatment cost on patients and the costs of providing long-term care for chronic patients $^{7-10}$, such as children with a disability ${ }^{11,12}$ or the elderly, there is no literature on the burden of in-hospital informal caregivers who care for adults who had served as the primary household income earner up until their injury. Here we define informal caregivers (hereafter: "caregivers") as family, friends, neighbors, or others who provide support for dependents and receive no remuneration for the help they provide ${ }^{10}$.

In Tanzania and other LMICs, caregivers play an important role in caring for hospitalized patients, including preparing food, changing dressings, assisting with medication administration, maintaining bedding, and other forms of support, all of which require a significant investment of time spent in the hospital. Literature from high-income countries (HICs) suggests that upon beginning their caregiving role, caregivers have decreased quality of life, poor mental health, significant stress, and increased anxiety about finances due to the physical and psychological stressors of their role ${ }^{13}$. Thirtyeight percent of caregivers of chronic adult patients report moderate to high financial strain as a result of providing care, especially those who live far from the care recipient, or care for patients with osteoporotic femur fractures ${ }^{14}$. In LMICs, caregivers of patients with lower extremity fractures are also more likely to miss school as a result of their role ${ }^{6}$. While some literature exists on the economic burden to the patient of having a femur fracture, including income lost while in the hospital, income lost following discharge as a result of disability, and hospital expenses, no studies have yet examined the economic burden incurred by caregivers.

Given this literature gap, the purpose of this study is to measure the initial economic burden (hereafter: "economic burden") of femur fractures on caregivers incurred during the patient's early hospital course. We hypothesize that caregivers lose significant income during a patient's hospitalization, and that the magnitude is correlated with length of hospitalization.

\section{Methods}

This study was designed as a prospective observational economic analysis conducted at Muhimbili Orthopaedic 
Institute (MOI) in Dar es Salaam, Tanzania. MOI is a large primary and referral orthopaedic hospital that sees a high volume of traumatic fracture injuries. Institutional review board approval was obtained from University of California, San Francisco (UCSF) and MOI. Convenience sampling was used to enroll all skeletally mature patients who sustained open or closed femoral shaft fractures (AO/OTA type 32) managed with intramedullary nailing at MOI between June 2014 and September 2014 were considered eligible. Patients were excluded if they had an ipsilateral tibia fracture, preoperative evidence of infection at the surgical site, clinical or radiographic evidence of pathologic fracture, or prior surgery involving the affected femur. Written, informed consent was obtained from all patients upon admission and from all caregivers upon interview.

Caregivers for each patient provided demographic information: age, sex, occupation, and highest educational level. Caregivers were asked how many times they had visited the patient, how many hours per visit they spent directly or indirectly caring for the patient (e.g., preparing meals, running errands), how many hours per visit they spent in transit to and from the hospital, and how much their transportation to and from the hospital cost. Length of hospital stay and time to interview (number of days between patient admission and caregiver interview) were calculated for each patient.

To determine the economic burden incurred by a caregiver, productivity losses were calculated using the Human Capital Approach, which assumes the value of lost time is equivalent to the amount an employer would pay to replace the equivalent time ${ }^{15}$. Time was valued using standardized income data from the World Health Organization (WHO), ensuring equitable valuation of potential productivity foregone due to caregiving for the acutely injured, regardless of occupation. The gross national annual income per capita in Tanzania was reported to be $\$ 1,750$ USD in $2013^{16}$. Assuming an 8-hour workday and a 5-day work week, the WHO standardized hourly wage was calculated to be $\$ 0.86 /$ hour. To assess lost wages, actual hours spent was first calculated by summing hours spent per visit and travel time per visit and multiplying the result by the number of visitations to date (at the time of interview) (Equation 1). To determine actual cost incurred to date, the actual hours spent was then multiplied by standardized WHO hourly wage data ${ }^{16}$ and the result added to the product of the travel cost per visit and the number of visitations to date (Equation 2). The cost per hospital day was then calculated by dividing the actual cost incurred by the time to interview (Equation 3).

To determine the total cost incurred by caregivers over the entire patient hospitalization, the cost per hospital day was multiplied by the length of stay (Equation 4). To determine the percent of the caregivers' annual income lost, the total cost incurred by a caregiver was divided by the standardized WHO annual income for Tanzania ${ }^{16}$ (Equation 5).

Data were transferred to Stata (Statacorp, College Station, TX) and descriptive statistics were used to report all data. Dichotomous variables were summarized as counts or proportions, and continuous variables were described by mean and standard deviation. Linear regression was used to assess the relationship between actual caregiver cost and number of patient days in hospital during time to interview. Significance was set at an alpha of less than 0.05. Economic figures are reported in 2014 U.S. dollars (USD).

\section{Table 1. Subject Demographics}

\begin{tabular}{lll} 
& Patients & Caregivers \\
$\mathrm{N}$ & 46 & 70 \\
Age, mean (SD) & $33.76(14.94)$ & $33.33(10.57)$ \\
Sex & & \\
$\quad$ Female & $9(20 \%)$ & $44(63 \%)$ \\
Male & $37(80 \%)$ & $26(37 \%)$ \\
Education & & \\
$\quad$ Primary & $3(7 \%)$ & $40(57 \%)$ \\
Secondary (0) & $24(52 \%)$ & $11(16 \%)$ \\
Secondary (A) & $11(24 \%)$ & $8(11 \%)$ \\
University or Higher & $5(11 \%)$ & $11(16 \%)$ \\
$\quad$ None & $3(7 \%)$ & $0(0 \%)$ \\
Employment & & \\
Employed & $37(80 \%)$ & $51(73 \%)$ \\
Works Within Home & $1(2 \%)$ & $11(16 \%)$ \\
$\quad$ Unemployed & $4(9 \%)$ & $3(4 \%)$ \\
Student & $4(9 \%)$ & $5(7 \%)$ \\
\hline
\end{tabular}

Table 2. Burden incurred by caregivers of femur fracture patients

\begin{tabular}{lccccc} 
& Mean & Median & Minimum & Maximum & Standard Deviation \\
Hours per visit $(\mathrm{hrs})$ & 5.12 & 3.00 & 1.00 & 13.50 & 4.30 \\
Travel time per visit $(\mathrm{hrs})$ & 3.65 & 4.00 & 0.67 & 6.67 & 1.71 \\
No. of visits & 9.10 & 5.00 & 1.00 & 41.00 & 8.69 \\
Actual Hours Spent $(\mathrm{hrs})$ & 82.11 & 39.25 & 4.00 & 480.00 & 100.57 \\
Travel cost per visit $\left(\$^{\mathrm{a}}\right)$ & 0.83 & 0.46 & 0.00 & 6.92 & 0.97 \\
Actual Cost $\left(\$^{\mathrm{a}}\right)$ & 79.56 & 46.72 & 3.44 & 426.63 & 95.10 \\
Time to Interview (days) & 13.34 & 6.50 & 1.00 & 57.00 & 13.29 \\
Cost per Hospital Day $\left(\$^{\text {a/day) }}\right.$ & 7.43 & 5.76 & 0.23 & 22.02 & 5.17 \\
& & & & & \\
Length of Stay (days) & 20.49 & 11.00 & 4.00 & 87.00 & 17.89 \\
Total Cost of Stay $\left(\$^{\mathrm{a}}\right)$ & 149 & 65.07 & 4.82 & 1225.62 & 227.54 \\
\hline \% Annual Income Lost & 9 & 3.72 & 0.28 & 70.04 & 13.00 \\
\hline
\end{tabular}




\section{Results}

Forty-six patients were enrolled in the study, and 73 caregivers were interviewed from a total of 84 unique caregivers $(86.9 \%$ interviewed). On average, each patient had between one and two (1.83) unique caregivers. The average age of caregivers was 33.3 years, and $44(63 \%)$ were female. Forty $(57 \%)$ had primary school education, $11(16 \%)$ had some secondary school-education (O-level), $8(11 \%)$ had secondary schooleducation (A-level), and $11(16 \%)$ had university education or higher. Fifty-one $(73 \%)$ caregivers were employed, 11 $(16 \%)$ worked within the home, $3(4 \%)$ were unemployed, and $5(7 \%)$ were students [Table 1]. The majority of caregivers $(89 \%)$ used bus or train as their primary mode of transportation to the hospital, with the remainder using motor bike $(4 \%)$ or car $(7 \%)$. Caregivers' average time spent per visit was 5.12 hours and average total travel time per visit was 3.65 hours, summed for an average total of 8.77 hours per visit. The average travel cost per visit was $\$ 0.83$. The average caregiver cost per hospital day was $\$ 7.43$. The patients' average length of stay for this hospitalization was 20.49 days. The average total caregiver economic burden over the entire length of hospitalization was $\$ 149$ and the average percent of annual income lost by caregivers was $9 \%$ [Table 2].

The cost incurred by caregivers was significantly correlated with patient time in hospital. Each additional day was associated with $\$ 4.77$ of incremental cost to the caregiver $(p<0.01)$ and time in hospital accounted for $45 \%$ of the explained variability in cost incurred [Figure 1].

\section{Discussion}

In this study, we used observation and interviews to estimate the economic burden of caregiving for hospitalized, adult patients admitted for intramedullary nailing of femoral shaft fractures in a LMIC setting. To our knowledge, this is the first such study. We found that on average, every such patient receives care from between 1-2 caregivers, who lose an average of $\$ 149$, or $9 \%$ of their annual income during a patient's hospital stay. This total economic burden correlated with the time a patient spent in the hospital, both leading up to and following definitive surgical fixation.

The most common profile for a caregiver was a 33-year-old female with primary school education who is self-employed or works within the home. This is similar to the profile of caregivers documented in previous studies-primarily middle-aged females with some education who are selfemployed ${ }^{17}$. A meta-analysis conducted by Thrush et al. on the burdens experienced by caregivers in LMIC settings found that the median age of caregivers was $45.1,74 \%$ were female, $66.6 \%$ completed some or all of primary school, and $54.5 \%$ were self-employed ${ }^{18}$. To capture the economic value of work within the home, and a wide variety of employment types, we utilized standardized WHO wage data for Tanzania. Prior research examining informal caregiving in LMICs has focused on at-home caregiving in the setting of chronic conditions like stroke, dementia, and HIV/AIDS ${ }^{19-21}$. This study instead focuses on the implication of extensive caregiving in the acute setting of orthopaedic injury, where caregivers must travel to the hospital frequently and spend extended periods of time supporting the patient. Caregivers spent, on average, more than the equivalent of a full workday on each visit when incorporating time spent in transit and time spent in the hospital. Though the ranges of actual time spent and resulting costs incurred by caregivers were wide, nearly twenty-five percent of the caregivers interviewed incurred burdens over the catastrophic expenditure threshold commonly recognized as $10 \%$ of annual income ${ }^{22}$. This is in addition to the direct medical and productivity losses of the injured patient ${ }^{23}$. In a prior study, we estimated the direct medical costs of a femur fracture were $\$ 531^{23}$; therefore caregiver costs represent $28 \%$ of direct medical costs. This cost directly correlates with time in hospital.

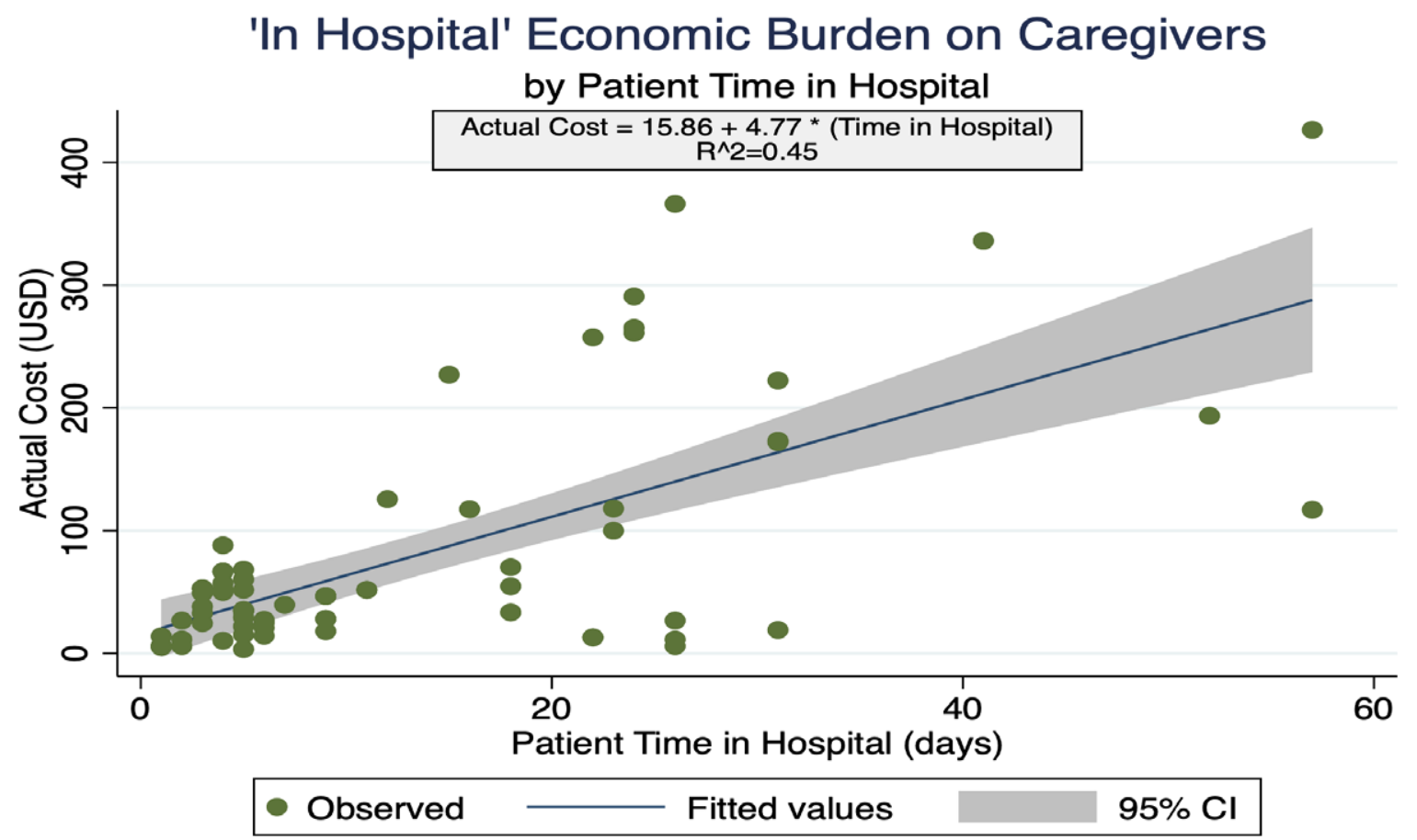

Figure 1. Economic burden, in USD\$, incurred by caregivers of patients with femur fractures over time in hospital, in days. Results of linear regression analysis shown in box, observed values are plotted as green dots, fitted regression line plotted in blue with $95 \%$ confidence interval plotted in gray. 
When placed in a broader economic context, Conway et al. found the femoral shaft fracture incidence across multiple hospitals in Tanzania to range from 2.1-18.4 per 100,000 people annually ${ }^{24}$. Using the 2014 World Bank population reported for Tanzania of 49,959,822 $2^{25}$, our findings of 1.83 caregivers per femur fracture patient and $\$ 149$ incurred per caregiver, we estimate the annual lost income and expense from caregiver responsibility throughout Tanzania to be $\$ 285,768-\$ 2,503,874$. Hence, efforts to reduce time to surgery by increasing surgical capacity have the potential to substantially decrease caregiver burden, thereby decreasing the overall economic impact of traumatic femur fractures.

There were several limitations to this study. First, the study was conducted at only one location, which may limit the generalizability. Second, our study focused on the initial economic burden incurred during the patient's hospital course; we did not attempt to measure the economic burden generated by sequelae from this initial period including but not limited to further costs associated with home care after patient discharge, sacrifice of income-generating assets for medical bill payment, and caregiver unemployment. Our estimation of the burden is therefore conservative and represents the minimum burden created by this event. Third, our estimation of caregiver burden rate (\$/day) assumes that caregiver visiting patterns remained constant throughout the patient's hospital course. This is likely to be a valid assumption as caregivers in this setting provide services such as housekeeping, toileting, and meal preparation that are necessary throughout the hospital stay. Fourth, the use of standardized wage data, though it ensures equitable valuation of many different kinds of time spent, may undervalue the impacts of stated costs, such as travel costs, on caregivers who do not earn money as a result of working within the home, being students, or being unemployed. Finally, we were not able to interview every caregiver for each patient due to time limitations during the capture period and because several caregivers declined to participate in the study. Despite this, our response rate was high, and these prospectively collected data provide important and novel information that build a foundation for more robust estimates of indirect costs associated with road traffic injuries resulting in skeletal trauma in LMICs.

Informal caregivers of patients treated operatively for femur fractures in this study lost an average of $\$ 149$, representing $9 \%$ of annual income. Costs incurred were positively correlated with patient time in hospital. A cost analysis of caregivers who provide aid to femur fracture patients expands our understanding of the economic and social burden of musculoskeletal injury in Tanzania and can inform future health policy implementation, funding and research.

\section{References}

1. Chandran A, Hyder AA, Peek-Asa C. The global burden of unintentional injuries and an agenda for progress. Epidemiol Rev 2010;32(1):110-20.

2. Gosselin RA, Spiegel DA, Coughlin R, Zirkle LG. Injuries: The neglected burden in developing countries. Bull World Health Organ 2009;87(4):246

3. Phillips J, Jergesen HE, Caldwell A, Coughlin R. IGOT-the institute for global orthopaedics and traumatology: A model for collaboration and change. Tech Orthop 2009;24(4):308-11.

4. Bach O. Musculo skeletal trauma in an East African public hospital. Injury 2004;35(4):401-6.
5. Agarwal-Harding KJ, von Keudell A, Zirkle LG, Meara JG, Dyer GSM. Understanding and Addressing the Global Need for Orthopaedic Trauma Care: J Bone Jt Surg 2016;98(21):1844-53.

6. O'Hara NN, Mugarura R, Potter J, Stephens T, Rehavi MM, Francois $\mathrm{P}$, et al. Economic loss due to traumatic injury in Uganda: The patient's perspective. Injury 2016;47(5):1098-103.

7. Arango-Lasprilla JC, Nicholls E, Cabrera TV, Drew A, JimenezMaldonado M, Martinez-Cortes ML. Health-related quality of life in caregivers of individuals with traumatic brain injury from Guadalajara, Mexico. J Rehabil Med 2011;43(11):983-6.

8. Liu H-Y, Yang C-T, Cheng H-S, Wu C-C, Chen C-Y, Shyu Y-IL. Family caregivers' mental health is associated with postoperative recovery of elderly patients with hip fracture: A sample in Taiwan. J Psychosom Res 2015;78(5):452-8.

9. Alfaro N, Lázaro P, Gabriele G, Garcia-Vicuña R, Jover JÁ, Sevilla J. Perceptions, Attitudes and Experiences of Family Caregivers of Patients With Musculoskeletal Diseases: A Qualitative Approach. Reumatol Clínica Engl Ed 2013;9(6):334-9.

10. Evercare and National Alliance for Caregiving. Family Caregivers - What They Spend, What They Sacrifice. Minnetonka, MN: Evercare \& Bethesda, MD: NAC, 2007.

11. Mugoya GCT, Mumba MN, Hooper LM, Witte T, Youngblood M. Depression and Intimate Partner Violence among Urban Kenyan Caregivers of Children with Disabilities. J Psychiatr Ment Health Nurs 2019;(205):jpm.12550.

12. Mobarak R, Khan NZ, Munir S, Zaman SS, McConachie H. Predictors of Stress in Mothers of Children With Cerebral Palsy in Bangladesh. J Pediatr Psychol 2000;25(6):427-33.

13. Moradi A, Ebrahimzadeh MH, Soroush MR. Quality of life of caregiver spouses of veterans with bilateral lower extremity amputations. Trauma Mon 2015;20(1):26-31.

14. Siddiqui MQA, Koh J, Howe T Sen, Sim L, Fook-Chong S, Tan C. Stress levels amongst caregivers of patients with osteoporotic hip fractures - A prospective cohort study. Ann Acad Med Singapore 2010;39(1):38-42.

15. Drummond MF, Sculpher MJ, Torrance GW, O’Brien BJ, Stoddart GL. Methods for the Economic Evaluation of Health Care Programmes. Oxford University Press; 2005.

16. World Health Organization. WHO | United Republic of Tanzania [Internet]. WHO; c2019 [cited 2019 Aug 23]. Available from: https:// www.who.int/countries/tza/en/.

17. Kipp W, Tindyebwa D, Rubaale T, Karamagi E, Bajenja E. Health Care for Women International Family Caregivers in Rural Uganda: The Hidden Reality. 2007;

18. Thrush A, Hyder A. The neglected burden of caregiving in low- and middle-income countries. Disabil Health J 2014;7(3):262-72.

19. Chepngeno-Langat G, Falkingham J, Madise NJ, Evandrou M, Chepngeno G. Socioeconomic Differentials Between HIV Caregivers and Noncaregivers: Is There a Selection Effect? A Case of Older People Living in Nairobi City Slums. Res Aging 32(1):67-96.

20. Mcgrath JW, Ankrah EM, Schumann DA, Nkumbi S, Lubega M. AIDS and the urban family: Its impact in Kampala, Uganda. AIDS Care 1993;5(1):55-70.

21. Heymann J, Kidman R. HIV/AIDS, declining family resources and the community safety net. AIDS Care - Psychol Socio-Med Asp AIDSHIV 2009;21(SUPPL. 1):34-42.

22. Layard R, Mayraz G, Nickell S. The marginal utility of income. J Public Econ 2008;92(8-9):1846-57.

23. Kramer EJ, Shearer DW, Marseille E, Haonga B, Ngahyoma J, Eliezer E, et al. The Cost of Intramedullary Nailing for Femoral Shaft Fractures in Dar es Salaam, Tanzania. World J Surg 2016;40(9):2098108. 
24. Conway D, Albright P, Eliezer E, Haonga B, Morshed S, Shearer DW. The burden of femoral shaft fractures in Tanzania. Injury 2019;50(7):1371-5.
25. The World Bank. Tanzania | Population, total | Data [Internet]. c2019 [cited 2019 Sep 12]. Available from: https://data.worldbank.org/ indicator/SP.POP.TOTL?locations=TZ. 